\title{
Construction of the Porcine Endogenous Retrovirus Envelope Glycoprotein A and B Specific Antibody
}

\author{
Jung Eun Lee ${ }^{1}$, Gye-Woong Kim², Young Bong Kim ${ }^{1}$ and Hong Yang Park ${ }^{1 *}$ \\ ${ }^{1}$ Department of Animal Biotechnology, Konkuk University, Seoul, Korea \\ ${ }^{2}$ Department of Animal Resource Science, College of Industrial Science, Kongju National University, \\ Kongju, Korea
}

\begin{abstract}
Xenotransplantation using porcine organs could potentially associate with the risk of pathogenic infections, because human tropic porcine endogenous retrovirus (PERV) particles could be released from pig cells or organs. While there is no evidence of PERV transmission to human, safety issues become a paramount concern. For the prevention of this transmission, specific immunological tools must be provided for PERV transmission detection. In this study we described the expression of PERV envelope proteins and the production of a specific antibody against PERV envelope (Env) glycoprotein. The nucleotide sequence harboring the partial region of glycoprotein 70 was cloned into the pET vector and envelope protein was expressed in E. coli. Approximately $42 \mathrm{kDa}$ recombinant Env protein (PERV Env-aa357) was purified by the Ni-affinity column. For antibody production, mice were immunized with the recombinant PERV Env-aa357. The generated anti-serum was tested using Western blot and immunocytochemical assay. We found that anti-PERV Env serum displayed the specificity against the PERV Envs (PERV-A and PERV-B) expressed not only in E. coli but also in mammalian cells, and PERV particles within the porcine cell lines (PK 15 and PK-1). Taken together, PERV antibody could be useful for detecting PERV infection or xenotransplantation transmission.
\end{abstract}

Key Words: PERV, Xenotransplantation, Envelope protein, Antibody

\section{INTRODUCTION}

Usage of porcine organs could be a solution for an insufficient organ supply in transplantation. However, there are some obstacles for xenotransplantation (1). Not only the immunological rejection between pig and human, but

Received: May 6, 2009/ Revised: June 4, 2009

Accepted: June 8, 2009

* Corresponding author: Hong Yang Park. Department of Animal Biotechnology, Konkuk University, Seoul 143-701, Korea.

Phone: +82-2-450-4208, Fax: +82-2-455-1044 e-mail: kimera@konkuk.ac.kr

** This work was supported by a research grant from the Ministry of Education, Science and Technology (Grant Number R01-2007-00021040-0), BioGreen 21 Program in Rural Development Administration and an Agricultural R\&D Promotion Center (20070501034009), and Ministry for Agriculture, Forestry and Fisheries, Republic of Korea (608001-5). also infection associated with porcine endogenous viruses (PERVs) naturally existing in the porcine genome is a major concern in xenotransplantation. PERVs have the ability to infect several human cell lines and the possibility of human transmission (2 4).

PERVs have been classified to the retroviral $\beta$ and $\gamma$ genera $(4,5)$. Infectious human tropic PERVs have been assigned to the PERV $\gamma 1$ group consisting of the subgroups $\mathrm{A}, \mathrm{B}$ and $\mathrm{C}$ according to env sequences $(5,6)$. PERV infectivity is determined by the functional antigenic epitopes on the surface of viral envelope proteins $(7,8)$. These are also responsible for mediating retroviral entry that involves a series of complex event including receptor bindings, conformational changes, and membrane fusion between virus and host cells. PERV-A and PERV-B showed infectivity in the cells of humans, pigs, and some other species 
in vitro, whereas PERV-C infected only pig cells (2, 9, $10 \sim 12$ ). A recently isolated PERV-A/C recombinant (PERV$\mathrm{NIH})$ has highly infectious human tropic characteristics; it comprises an env gene with PERV-A in the surface glycoprotein region and PERV-C in the $\mathrm{C}$-terminal residues of the transmembrane region $(4,13 \sim 15)$.

PERV envelope glycoproteins consist of gp70 and transmembrane domain (p15E) derived from intracellular proteolytic processing of the Env precursor protein (16). The $\mathrm{N}$-terminal domain of gp70 is responsible for binding the virus to host cell receptor $(7,17)$ and conserved C-terminal region contains a transmembrane domain that is required for viral entry (18). The gp70 component undergoes major conformational rearrangement after receptor binding, allowing the $\mathrm{p} 15 \mathrm{E}$ protein to trigger viral and cytoplasmic membrane fusion.

To study PERVs in xenotransplantation, antibody for PERV detection is critical for prevention and monitoring of PERV transmission. However, there is no commercial supplier who released the antibodies product. Thus, we tried to construct and characterize the anti-sera specific for PERV envelope gp70, which has many potentials as a tool for monitoring of PERV infection.

\section{MATERIALS AND METHODS}

\section{Cells}

HeLa, porcine kidney cell lines, PK-15 (ATCC CCL33), LLC-PK1 (ATCC CL-101), and human 293T cells were maintained in Dulbecco's modified Eagle's medium (DMEM) supplemented with 10\% fetal bovine serum (GIBCO BRL, Grand Island, NY, USA), 2 mM L-glutamine, $50 \mu \mathrm{g} / \mathrm{ml}$ penicillin and streptomycin (GIBCO BRL) at $37^{\circ} \mathrm{C}$ in a humidified $5 \% \mathrm{CO}_{2}$ incubator.

\section{Cloning and expression of the PERV envelope gene}

PERV-A env (aa357) was amplified from the previously constructed pGEM-T-Y9 clone (PERV envelope type A, DQ011706) by polymerase chain reaction using a specific primer set; forward primer: 5'-TTTGAATTCATATGCATCCCACGTTAAGCCGG-3' and reverse primer: 5'-GGAAC-
ATAGAGACCAATGTACACATCATCATCATCATCATTAGGTCGACTT-3'. The PCR product was digested with Nde I and Sal I within PCR primers, and the $1071 \mathrm{bp}$ fragment of PERV env was cloned into pET-23a $(+)$ expression vector (Novagen, Madison, WI, USA), and named pET-PERV env-aa357. The recombinant PERV Env protein was expressed in the E. coli BL21 (DE3) pLysS (Novagen). The protein expression was induced by addition of $1.0 \mathrm{mM}$ IPTG when the cell growth reached to the OD 0.5 . Thereafter, the cells were harvested after $16 \mathrm{hr}$ incubation at $30^{\circ} \mathrm{C}$.

\section{Purification of expressed PERV Env protein}

To purify the recombinant protein, bacterial cells transformed with pET-PERV env-aa357 were cultured in $200 \mathrm{ml}$ LB media at $30^{\circ} \mathrm{C}$, and harvested cells were resuspended with pH 8.0 lysis buffer $\left(100 \mathrm{mM} \mathrm{NaH}_{2} \mathrm{PO}_{4}, 10 \mathrm{mM}\right.$ Tris-Cl, $8 \mathrm{M}$ Urea) and disrupted by sonication (SONOPLUS BANDELIN, Berlin, Germany). The cleared lysates were mixed with $1 \mathrm{ml}$ of $50 \%$ Ni-NTA resin (QIAGEN, Valencia, CA, USA) and incubated at $4{ }^{\circ} \mathrm{C}$ for overnight with gentle agitation. Then, Ni-NTA agarose beads were packed in column and washed with pH 6.3 washing buffer $(100 \mathrm{mM}$ $\mathrm{NaH}_{2} \mathrm{PO}_{4}, 10 \mathrm{mM}$ Tris-Cl, $8 \mathrm{M}$ Urea, $20 \mathrm{mM}$ imidazole) 2 times. Finally, proteins were eluted with $\mathrm{pH} 4.8$ elution buffer (100 mM NaH $2 \mathrm{PO}_{4}, 10 \mathrm{mM}$ Tris-Cl, $8 \mathrm{M}$ Urea, 200 $\mathrm{mM}$ imidazole). The purified protein solution was concentrated with Centricon-30 (AMICON INC., Beverly, MA, USA). Final protein concentration was determined using serially diluted bovine serum albumin (Sigma, St. Louis, MO, USA).

\section{Immunization of mouse}

$\mathrm{Balb} / \mathrm{c}$ mice were immunized intraperitoneally three times for two weeks intervals using $50 \mu$ g of purified Env protein emulsified in complete Freund's adjuvant (Sigma). Blood was taken from the mice and the antibody was analyzed using Western blot.

\section{SDS-PAGE and immunoblot assay}

The PERV expressing E. coli lysates were analyzed on 
10\% SDS-PAGE gel and stained with Coomassie brilliant blue R250 dye. The PERV envelope proteins were transferred to a nitrocellulose membrane and incubated with each 1:5000 anti-His probe (Santa Cruz Biotechnology, Santa Cruz, CA, USA). After washing three times with TBSTween $0.1 \%$, the membrane was incubated with 1:5000 goat anti-rabbit IgG HRP conjugate for $1 \mathrm{hr}$. Subsequently, the membrane was developed with the chemiluminescence substrate solutions (PIERCE, Rockford, IL, USA). The polyclonal antibody from PERV Env-aa357 immunized mice was tested with the same way as previously described except the goat anti-mouse IgG HRP conjugate. PERV-A and PERV-B envelope proteins expressed from 293T were also analyzed with the mouse antiserum to the PERV Env.

\section{Immunocytochemistry}

Porcine cell lines and HeLa cells were seeded at $1 \times$ $10^{3}$ cells per each well with cover slips in the 4 well plates. After fixing with $3.5 \%$ formaldehyde at room temperature for $5 \mathrm{hr}$, cells were washed with PBS two times. To block

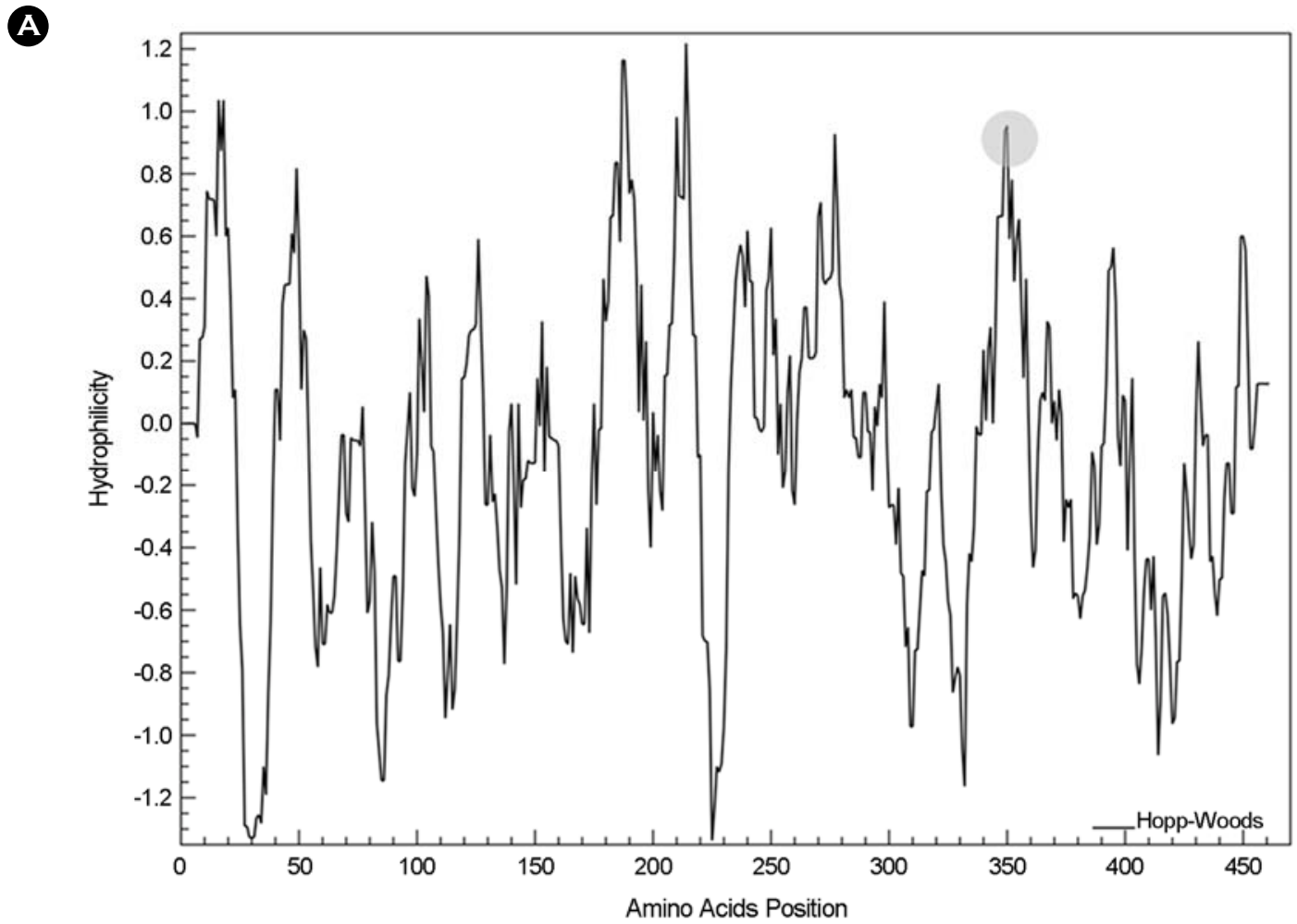

B

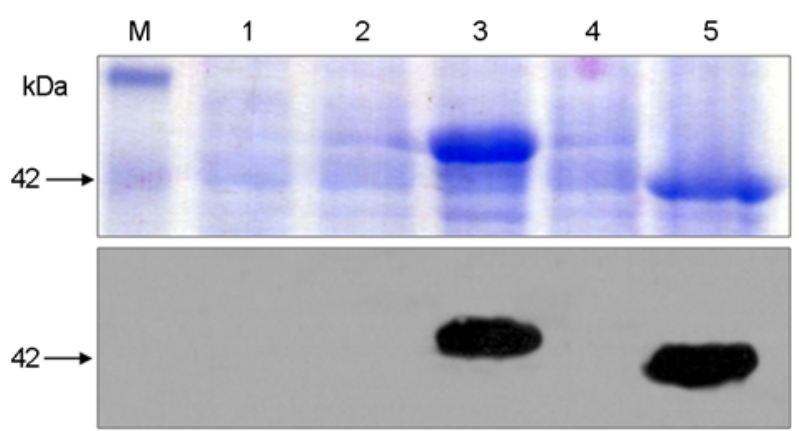

Figure 1. Expression of PERV envelope gp70 and Env-aa357. (A) Hydrophilicity of full length of PERV gp70 isolated from pig (DQ011706). The graph was drawn by Hoop-Woods method. The gray circle represents the hydrophilic C-terminal region of PERV Env-aa357. (B) SDS-PAGE (upper panel) and Western blot analysis (lower panel) of expressed PERV Envs. The expressed PERV Envs from E. coli were analyzed and stained with Coomassie blue (upper panel). Expressed recombinant proteins were confirmed with an anti-His antibody (lower panel). M, protein marker; lane 1, BL21 (DE3) pLysS; lane 2, pET-gp70 transformed cell lysate before induction; lane 3, pET-gp70 transformed cell lysate after induction; lane 4, pET-aa357 transformed cell lysate before induction; lane 5, pET-aa357 transformed cell lysate after induction. The arrow indicates molecular mass of aa357 protein, approximately $42 \mathrm{kDa}$. 
nonspecific binding, washed cells were incubated with 5\% normal goat serum in PBS for $30 \mathrm{~min}$. The cells were incubated with the anti-mouse IgG FITC-labeled secondary antibody (Santa Cruz Biotechnology) at room temperature for $1 \mathrm{hr}$. After three times of wash, the cells were covered in mounting medium (Vector Laboratories, Inc., Burlingame, CA, USA) and analyzed using the confocal laser scanning microscope (FV1000, Olympus, Tokyo, Japan) with appropriate filters.

\section{RESULTS}

\section{Expression of PERV recombinant envelope protein: PERV Env-aa357}

The full length of gp70 envelope gene originated from PERV Y9, DQ011706, isolated from Yorkshire, was cloned into pET-23a vector. Approximately $50 \mathrm{kDa}$ of gp70 Env protein had been expressed well in E. coli with body inclusion. However, it did not bind to the Ni-agarose resin efficiently. To improve protein purification efficiency by increasing solubility, amino acids sequences 1 to 357 were selected based on the hydrophilicity score using by HoppWoods method (Fig. 1A) (19). According to the sequence

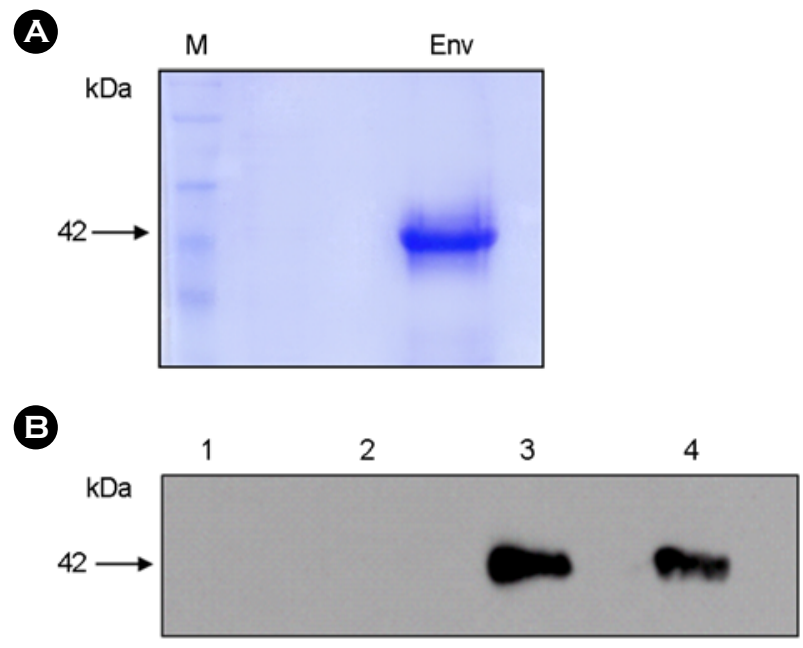

Figure 2. Purification of the recombinant PERV Env-aa357. Affinity purified Env protein $(\approx 50 \mu \mathrm{g})$ was analyzed on a $10 \%$ SDS-PAGE gel (A) and stained with Coomassie blue and confirmed with the mouse anti-PERV antibody (B). Lane 1, E. coli BL21 (DE3) pLysS; lane 2, pET-aa357 transformed cell lysate before induction; lane 3, pET-aa357 transformed cell lysate after induction; lane 4, purified PERV Env-aa357. analysis, truncated gp70 recombinant Env protein encoded by nucleotide $1071 \mathrm{bp}$ (357 amino acids) of Y9 with hydrophilic C-terminal domain was cloned into pET-23a vector and designated pET-PERV env-aa357. Recombinant expression vectors for pET-gp70 and pET-PERV env-aa357 were transformed into E. coli BL21 (DE3) pLysS and induced with IPTG stock at a final concentration $1.0 \mathrm{mM}$. Fig. 1B shows the PERV envelope glycoproteins expressed in E. coli BL21 (DE3) pLysS. Compared to full length of gp70, truncated gp70 recombinant Env (PERV Env-aa357) showed a molecular mass of $42 \mathrm{kDa}$ approximately. Expression of PERV Envs was confirmed by an anti-His antibody (Fig. 1B).

The expressed PERV Env-aa357 protein was purified by affinity chromatography using His-tag resin. The expression efficiencies of these two proteins ranged from $10 \sim 20 \%$ of the total cellular proteins. The purified PERV Env-aa357 was verified through the SDS-PAGE Coomassie blue staining and Western blot with an anti-PERV antibody, respectively (Fig. 2).

\section{Reactivity of antibody against PERV Env protein}

Anti-PERV Env antibody was prepared by immunizing female Balb/c mice with the purified PERV protein. Immunization was carried out with $50 \mu \mathrm{g}$ of the purified PERV Env-aa357 protein intraperitoneally. For boosting of antibody, second and third immunizations were carried out with three week interval. Three days after the final immunization, mice

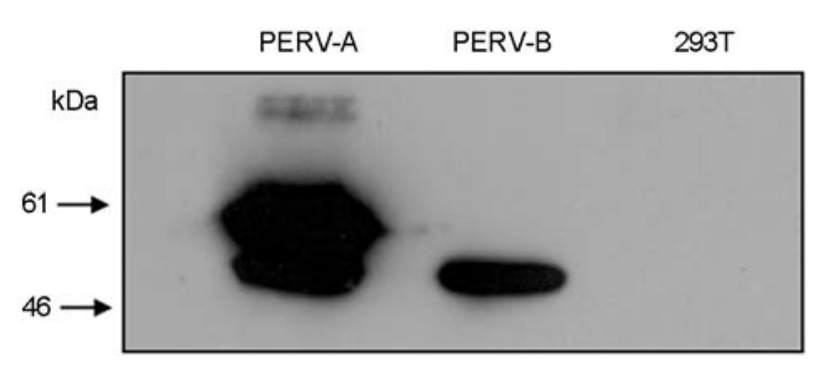

Figure 3. Western blot analysis of the constructed anti-PERV antibody with PERV gp70 envelope proteins expressed in mammalian cells. The two types of PERV-A and -B envelope glycoprotein constructs were expressed in the 293T cells and those cell proteins were analyzed with the constructed anti-PERV antibody. PERV proteins originated from $293 \mathrm{~T}$ cells were expressed by a previous method using vaccinia expression system. 

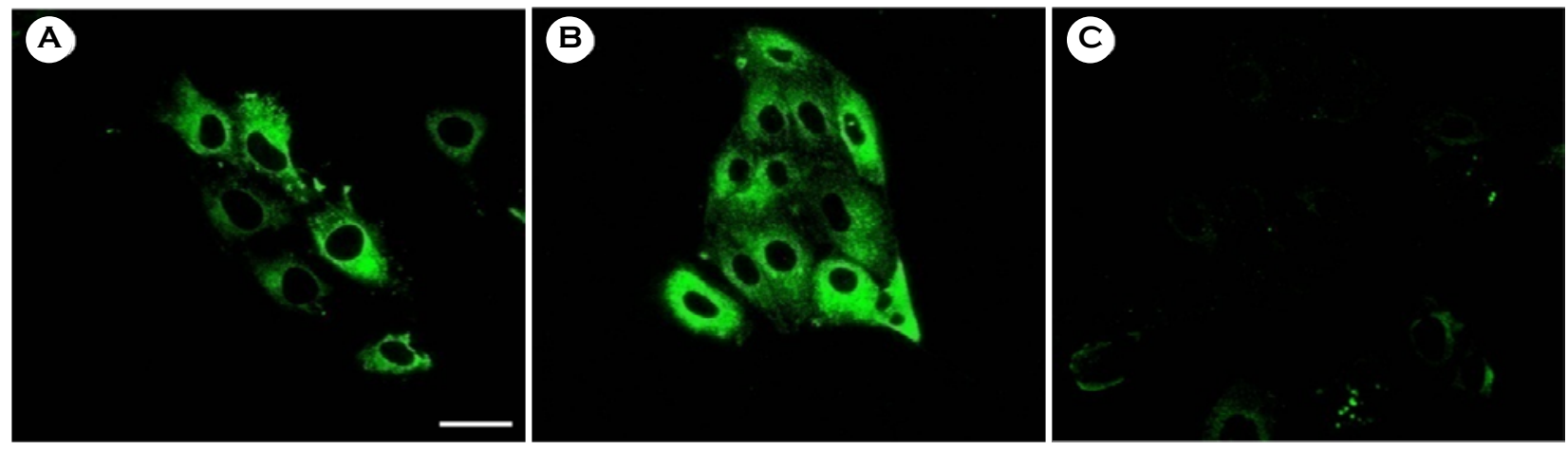

Figure 4. Immunocytochemical staining of the PERV producing porcine cells with constructed anti-PERV antibody. Porcine cells were stained with the constructed anti-PERV antibody and FITC conjugated anti-mouse secondary antibody, and visualized by confocal laser microscopy. FITC staining was measured at $488 \mathrm{~nm}$ and non-specific fluorescence measured at $543 \mathrm{~nm}$ was subtracted. Indirect immunofluorescence was visualized with an Olympus confocal microscope FV1000 at a magnification of $60 \mathrm{X}$. PK15 cell (A), PK-1 cell (B), and HeLa cell (C). The scale bar represents $30 \mu \mathrm{m}$.

bloods were collected. The specificity of the anti-PERV antibody was determined by Western blot analysis using bacterial cell and human 293T cell lysates expressing the recombinant PERV Env-aa357 protein in prokaryotic and PERV-gp70 in eukaryotic system, respectively. Antibody reactivity against the eukaryotic antigen is very important for detecting authentic viruses because PERV envelope protein undergoes post-translational modification such as glycosylation. To determine antibody activity against PERV Env expressed in mammalian cells, the recombinant gp70 of PERV-A and -B protein produced in 293T cells via recombinant vaccinia system (20) were examined (Fig. 3). By an immune blot analysis, anti-PERV Env-aa357 antibody produced from mice showed specific reactivity against PERV Envs expressed not only in prokaryotic but also in eukaryotic system.

\section{Indirect immunofluorescence staining of PERV producing porcine cells}

To determine antibody reactivity against PERV particles produced in pig cells, immunocytochemical staining was carried out with the constructed mouse PERV Env antibody. The localization of PERV Env protein on each cell line was visualized under the confocal microscope. PERV specific immunofluorescence (IF) signals were detected with the mouse anti-PERV Env antibody, revealing Env-associated fluorescence signals (Fig. 4). Strong IF signals were observed in two porcine cell lines with similar patterns, while $\mathrm{HeLa}$ showed very weak signals that were thought to be nonspecific binding.

\section{DISCUSSION}

Recently, PERV detection system based on an antibody generated against synthetic peptides has been reported (6) and was demonstrated to be highly sensitive and specific to PERV Gag protein comprising large part of the PERV viral particle $(6,21 \sim 23)$. The anti-Env antibody was employed to detect PERV infection. However the antibody to Env protein, attained smaller part in virus than Gag, is apparently not sensitive enough to explain the presence of the virus in the cells. Moreover, the elucidation of the cleavage status of the envelope proteins using the previous Env antibody is unclear. Even if the signals were appeared, they are very weak or with nonspecific bands in immunoblotting assays $(6,21,23)$. Under these environments, we chose the gp70 domain for the generation of specific antibody. However, the expressed gp70 was not bound to the Ni-NTA resin efficiently. To purify the PERV Env protein using affinity chromatographic method, we removed the C-terminal hydrophobic region of PERV gp70. Truncated gp70 was generated by removing C-terminal 98 amino acids. Thus, truncated gp70 showed improved purification efficiency than intact form. 
Env-aa357 protein was purified efficiently by deleting the hydrophobic region of gp70 for improving solubility and then expressed to $42 \mathrm{kDa}$ in prokaryotic cells. Purification was carried out in denaturing condition. Mice were immunized with this purified protein, to generate the specific antibody. The constructed PERV Env antibody was tested using Western blot with PERV Envs expressed in E. coli as well as human 293 T cells. Fig. 3 supports that the mouse anti-PERV antibody has the specificity against PERV-A and -B Envs expressed in human 293T cells. The gp70 and truncated gp70 regions of PERV-A and -B amino acid sequences showed differences with $37.8 \%$ and $39.5 \%$, respectively. Surprisingly, our constructed PERV antibody could detect the both types of PERV Env proteins. Thus, this implies another human tropic $\mathrm{A} / \mathrm{C}$ recombinant PERV can be detected by the constructed anti PERV antibody.

Our constructed anti PERV antibody could react to PERV Env protein and had the sensitivity to detect viral particles in pig cells. The antibody specificity is enough to perform the immunocytochemical assay. Although monoclonal antibody construction is required for more increased specificity, considering that human tropic PERVs are PERV subgroup $\mathrm{A}$ and $\mathrm{B}$, constructed antibody in this study is directly applicable to the detection of viral protein expression in PERV infection as well as PERV transmission on xenotransplantation.

\section{REFERENCES}

1) Cooper DK. Clinical xenotransplantion--how close are we? Lancet 2003;362:557-9.

2) Patience $C$, Takeuchi $Y$, Weiss RA. Infection of human cells by an endogenous retrovirus of pigs. Nat Med 1997;3:282-6.

3) Ritzhaupt A, Van Der Laan LJ, Salomon DR, Wilson CA. Porcine endogenous retrovirus infects but does not replicate in nonhuman primate primary cells and cell lines. J Virol 2002;76:11312-20.

4) Wilson CA, Wong S, Muller J, Davidson CE, Rose TM, Burd P. Type $\mathrm{C}$ retrovirus released from porcine primary peripheral blood mononuclear cells infects human cells. J Virol 1998; 72:3082-7.
5) Blusch JH, Patience C, Martin U. Pig endogenous retroviruses and xenotransplantation. Xenotransplantation 2002;9:242-51.

6) Galbraith DN, Kelly HT, Dyke A, Reid G, Haworth C, Beekman J, Shepherd A, Smith KT. Design and validation of immunological tests for the detection of porcine endogenous retrovirus in biological materials. J Virol Methods 2000;90:115-24.

7) Akiyoshi, DE, Denaro M, Zhu H, Greenstein JL, Banerjee P, Fishman JA. Identification of a full-length cDNA for an endogenous retrovirus of miniature swine. J Virol 1998;72:4503-7.

8) Le Tissier P, Stoye JP, Takeuchi Y, Patience C, Weiss RA. Two sets of human-tropic pig retrovirus. Nature 1997;389:681-2.

9) Martin U, Kiessig V, Blusch JH, Haverich A, von der Helm K, Herden T, Steinhoff G. Expression of pig endogenous retrovirus by primary porcine endothelial cells and infection of human cells. Lancet 31998;352: 692-4.

10) Martin U, Winkler ME, Id M, Radeke H, Arseniev L, Takeuchi Y, Simon AR, Patience C, Haverich A, Steinhoff G. Productive infection of primary human endothelial cells by pig endogenous retrovirus (PERV). Xenotransplantation 2000;7:138-42.

11) Specke V, Tacke SJ, Boller K, Schwendemann J, Denner J. Porcine endogenous retroviruses: in vitro host range and attempts to establish small animal models. J Gen Virol 2001;82:837-44

12) Takeuchi Y, Patience C, Magre S, Weiss RA, Banerjee PT, Le Tissier P, Stoye JP. Host range and interference studies of three classes of pig endogenous retrovirus. J Virol 1998;72:9986-91.

13) Bartosch B, Stefanidis D, Myers R, Weiss R, Patience C, Takeuchi Y. Evidence and consequence of porcine endogenous retrovirus recombination. J Virol 2004;78: 13880-90.

14) Harrison I, Takeuchi Y, Bartosch B, Stoye JP. Determinants of high titer in recombinant porcine endogenous retroviruses. J Virol 2004;78:13871-9.

15) Scobie L, Taylor S, Wood JC, Suling KM, Quinn G, Meikle S, Patience C, Schuurman HJ, Onions DE. Absence of replication-competent human-tropic porcine endogenous retroviruses in the germ line DNA of inbred 
miniature Swine. J Virol 2004;78:2502-9.

16) Pinter A, Kopelman R, Li Z, Kayman SC, Sanders DA. Localization of the labile disulfide bond between SU and TM of the murine leukemia virus envelope protein complex to a highly conserved CWLC motif in SU that resembles the active-site sequence of thiol-disulfide exchange enzymes. J Virol 1997;71:8073-7.

17) Best S, Le Tissier PR, Stoye JP. Endogenous retroviruses and the evolution of resistance to retroviral infection. Trends Microbiol 1997;5:313-8.

18) Gemeniano M, Mpanju O, Salomon DR, Eiden MV, Wilson CA. The infectivity and host range of the ecotropic porcine endogenous retrovirus, PERV-C, is modulated by residues in the $\mathrm{C}$-terminal region of its surface envelope protein. Virology 2006;346:108-17.

19) Hopp TP, Woods KR. Prediction of protein antigenic determinants from amino acid sequences. Proc Natl Acad Sci USA 1981;78:3824-8.
20) Lee D, Lee J, Uhm SJ, Lee YS, Park MJ, Park HY, Kwon M, Lee HT, Kim YB. Molecular characterization of the porcine endogenous retrovirus subclass A and B envelope gene from pigs. Transplant Proc 2006;38:3066 -9 .

21) Fischer N, Krach U, Niebert M, Tonjes RR. Detection of porcine endogenous retrovirus (PERV) using highly specific antisera against Gag and Env. Virology 2003; 311:222-8.

22) Krach U, Fischer N, Czauderna F, Kurth R, Tonjes RR. Generation and testing of a highly specific anti-serum directed against porcine endogenous retrovirus nucleocapsid. Xenotransplantation 2000;7:221-9.

23) Tacke SJ, Bodusch K, Berg A, Denner J. Sensitive and specific immunological detection methods for porcine endogenous retroviruses applicable to experimental and clinical xenotransplantation. Xenotransplantation 2001; 8:125-35. 